\title{
Genetic Diversity Assessment of Ethiopian Landrace Barley (Hordeum Vulgare (L.)) Genotypes Through Multivariate Analysis
}

\author{
Delessa Angassa, Jemal Mohammed* \\ Crop and Horticulture Biodiversity Directorate, Ethiopian Biodiversity Institute, Addis Ababa, Ethiopia
}

Email address:

jemalm781@gmail.com (J. Mohammed)

${ }^{*}$ Corresponding author

\section{To cite this article:}

Delessa Angassa, Jemal Mohammed. Genetic Diversity Assessment of Ethiopian Landrace Barley (Hordeum Vulgare (L.)) Genotypes Through Multivariate Analysis. American Journal of Biological and Environmental Statistics. Vol. 7, No. 3, 2021, pp. 67-73.

doi: $10.11648 /$ j.ajbes.20210703.12

Received: August 10, 2021; Accepted: August 25, 2021; Published: September 7, 2021

\begin{abstract}
Assessing the level of genetic diversity of collected germplasms enable breeders to exploit existed genetic resources for further breeding programs. Therefore, one hundred thirty-eight Barley (Hordeum Vulgare (L.)) accessions and three standard checks were evaluated using augmented design in the 2019 main cropping season. The accessions were planted in un-replicated plots and the three standard checks were replicated once in each block. The PCA showed that the first two principal components contributed $67.68 \%$ of the total variations observed among the genotypes. Principal component one (PC1) alone had contributed $43.02 \%$ of the total variations mainly due to tillers per plant, spike length and thousand seed weight in their respective order. Principal component two (PC2) contributed $24.66 \%$ of the total variations mainly through days to $50 \%$ flowering, days to $90 \%$ maturity, and thousand seed weight in their descending order. Genotypes were grouped into four clusters where $51.3 \%, 33.3 \%$, $14.0 \%, 0.7 \%$ and $0.7 \%$ of genotypes fall in cluster I, II, III, and IV, respectively. The result revealed the existence of significant agro-morphological variations among the genotypes included in the study. Based on the characters considered and populations evaluated, the marked diversity observed among the barley genotypes in Ethiopia could be utilized in future crop improvement for various agronomically important traits. Generally, the study revealed the existence of wide range of diversity among barley accessions which has paramount importance for further exploitation of the important traits.
\end{abstract}

Keywords: Clustering, Diversity, Genotypes, Multivariate, Principal Component, Quantitative Traits

\section{Introduction}

Barley (Hordeum vulgare L.) belongs to family Poaceae and has been cultivated for the last many years in Ethiopia. Ethiopia is considered as one of the Vavilov's center of diversity for barley because of high levels of genetic and phenotypic diversity and strong genetic differentiation from Asian and North African Populations [1] due to the presence of variation in altitude, soil types, climate, topography and geographical isolation for the long period [2].

In Ethiopia, barley is among the main cereal crops and ranks fifth after teff, maize, sorghum and wheat in terms of area and maize, teff, wheat and sorghum in terms of production. During 2019/20 growth season, 950,742.01 hectares of land was covered by barley in farmers' farms in Ethiopia with a total harvest of 2,378,010.2 tons with the productivity of $25.01 \mathrm{qt} / \mathrm{ha}$. It accounts for about $7.39 \%$ and $7.09 \%$ of the total national cereal area and production, respectively [3].

Barley is used as a food and beverage in more than 20 different ways in the country. Bread and Injera (pancake-like bread) are among the major types of barley products to consume. Roasted or cooked grain is also consumed alone or mixed with beans and peas. The powder is made to porridge either boiled or raw. Kinche (a type of bulgur) is another type of food prepared from semi-milled grains of barley [4].

Ethiopian Biodiversity Institute collected about 17,000 accessions from barley growing regions of the country which covers a wide range of agro-ecological conditions and is conserved in the national gene bank. Even though the collections are numerous with various characters which may 
be different or interrelated, the magnitude of the variation is not yet properly studied and documented. Assessing the variability and grouping the existed accessions based on morphological characters enable breeders to exploit existed genetic resources for further breeding programs. It is also important to know the major traits contributing to the total observed variations among the farmers' varieties accessions. Therefore, this study aimed to assess the diversity and estimate the magnitude of genetic distance and identify the major traits contributing to the observed variations among barley accessions using multivariate analysis.

\section{Materials and Methods}

\subsection{Description of the Study Area}

The experiment was conducted at Ethiopian Biodiversity Institute Arsi Negelle research site, West Arsi zone of Oromia region during 2019 main cropping season under rain fed condition. The research site is located $7^{\circ} 19^{\prime} 22^{\prime \prime} \mathrm{N}$ latitude and 38 39'20" E longitude with an altitude of $1933 \mathrm{~m}$ above sea level. The mean annual minimum and maximum 6.8 and $27.2^{\circ} \mathrm{C}$, respectively while rainfall varies 250 to $750 \mathrm{~mm}$ per annum. The soil of the experimental site was sandy loam [5].

\subsection{Experimental Materials and Design}

The plant materials used in this study consists of 135 barley accessions held in the Gene bank of Ethiopian Biodiversity Institute. Three released varieties (HB-1963, HB-1964 and IBON-173/03) obtained from the Kulumsa Agricultural Research Centre were included as standard checks and a total of 138 barley genotypes (accessions + standard checks) were used in the study.

The experiment was laid down in augmented design with no replication among the barley accessions and three standard checks replicated in every block. The genotypes were planted on 23 July 2019 with diammonium phosphate (DAP) at the rate of $100 \mathrm{~kg} \mathrm{ha}^{-1}$ and UREA at the rate of $80 \mathrm{~kg} \mathrm{ha}^{-1}$ (complete application at sowing) and other management practices were applied as recommended for the site. Each treatment was planted on $2 \mathrm{~m} \mathrm{x} 0.6 \mathrm{~m}\left(1.2 \mathrm{~m}^{2}\right.$ plot size and accommodated four rows of $2 \mathrm{~m}$ length with $20 \mathrm{~cm}$ distance. The outer most rows at both ends of plots were considered as borders. The second and third rows at one side of each plot were designated as sampling area. The distance between plots and blocks was $0.5 \mathrm{~m}$ and $1 \mathrm{~m}$ apart, respectively.

\subsection{Data Collection}

All field and laboratory data were recorded accordingly to the International Plant Genetic Resources Institute's barley descriptor list [6]. Data of nine quantitative traits were recorded on randomly selected and tagged 20 individual plants for the research from each plot and data were collected for number of tillers per plant, plant height, spike length and number of kernels per spike. Whereas, days to $50 \%$ flowering, days to $75 \%$ maturity, thousand seed weight and grain yield were recorded on plot bases.

\subsection{Statistical Analysis}

\subsubsection{Analysis of Variance}

Analysis of variance was analyzed for all agromorphology traits using $\mathrm{R}$ statistical software (version 4.0.4; augmented RCBD package) [7].

\subsubsection{Principal Component Analysis}

The data were standardized to mean zero and variance of one before computing principal component analysis to avoid differences in measurement scales. The principal component based on the correlation matrix was calculated using $\mathrm{R}$ statistical software (version 4.0.4; Facto Mine R package) [8].

\subsubsection{Euclidean Distance and Clustering of Genotypes}

Euclidean Distance (ED) was computed from all traits after standardization (subtracting the mean value and dividing it by the standard deviation) as established by Sneath and Sokal [9]. The distance matrix from phenotype traits was used to construct dendrogram based on the Unweighted Pair Group Method with Arithmetic Means (UPGMA). The results of the cluster analysis were presented in the form of dendrograms. $\mathrm{R}$ statistical software (version 4.0.4; factoextra package) [10] used for the analysis of distance matrix and constructing Dendrogram.

\section{Results and Discussion}

\subsection{Analysis of Variance}

The analysis of variance of the nine quantitative traits indicated the presence of significant variations at $(\mathrm{P}<0.01)$ between the tested genotypes for days to $50 \%$ flowering, number of tillers per plant, spikelet per spike and thousands seed weight. Traits such days to $75 \%$ maturity and grain yield were significant at $(\mathrm{P}<0.05)$. Plant height, spike length and kernels per spike were not seen significant differences between genotypes.

\subsection{Principal Component Analysis}

The PCA was performed to estimate the relative importance and contribution of each quantitative trait to the total variance and illustrate the agronomic diversity among the 138 barley genotypes.

Principal component analysis results from nine quantitative traits of 138 barley genotypes are presented in Table 1. The principal component analysis resulted in two principal components ( $\mathrm{PC} 1$ to $\mathrm{PC} 2$ ) with eigenvalues ranged from 2.22 to 3.87 . The two PCs were retained in the analysis because the two PC had an eigenvalue $\geq 1$. The PCs with an eigenvalue of $<1$ were ignored due to Gutten's lower bound principle that eigenvalues $<1$ should be ignored.

The first two principal components (PCs) with Eigenvalue $\geq 1$ accounted for $67.68 \%$ of the total variability. The first PC explained $43.02 \%$ of the variance. For principal component one (PC1), number of kernel per spike (0.46), spikelet per spike (0.45) and spike length (-0.40) have the higher 
eigenvector contribution. Therefore, most of the variations among genotypes in principal component one was brought due to three major traits indicated above. Principal component axis two (PC2) had contributed $24.66 \%$ of the total variation. The observed variations in $\mathrm{PC} 2$ were mainly brought by days to $75 \%$ maturity $(0.53)$ and days to $50 \%$ flowering (0.47) and grain yield (-0.48) eigenvector values.

In agreement with this study, Ebrahim [11] studied other 20 Ethiopian barley varieties from Wollo highlands and reported that the first three principal components together accounted for $84.22 \%$ of the total variations observed among the barley genotypes through kernel number per spike, kernel weight per spike, number of spikelet per spike, thousand seed weight, spike length, grain yield per plot, days to heading and maturity.

A study by Tarekegn [12] on another 20 Ethiopian landraces barley genotypes also reported that the first three principal components contributed $73.14 \%$ of the total variations contributed through productive tillers per plant, grain yield and thousand grain weight, spike length and awn length from the total genotypic variations observed. Similarly, Mekonnon et al. [13] also reported that the first four principal components were accommodated $72 \%$ of the variability. Days to $50 \%$ flowering, days to maturity, number of seed per spike and peduncle extension were the most contributing traits in the first principal component [13].

Table 1. Eigenvector of the nine principal components for traits of 138 barley genotypes.

\begin{tabular}{|c|c|c|c|c|c|c|c|c|c|}
\hline Traits & PC1 & PC2 & PC3 & PC4 & PC5 & PC6 & PC7 & PC8 & PC9 \\
\hline Days to $50 \%$ flowering & 0.30 & 0.47 & 0.11 & 0.04 & 0.26 & -0.04 & 0.28 & -0.73 & 0.01 \\
\hline Days to $75 \%$ maturity & 0.23 & 0.53 & -0.02 & -0.18 & 0.06 & -0.15 & 0.46 & 0.63 & -0.04 \\
\hline Plant height $(\mathrm{cm})$ & 0.26 & -0.27 & 0.42 & 0.75 & 0.01 & -0.22 & 0.23 & 0.14 & -0.01 \\
\hline Days to $75 \%$ maturity & 0.23 & 0.53 & -0.02 & -0.18 & 0.06 & -0.15 & 0.46 & 0.63 & -0.04 \\
\hline Number of tillers per plant & -0.38 & -0.19 & -0.31 & 0.19 & 0.18 & 0.49 & 0.65 & -0.03 & 0.01 \\
\hline Spike length (cm) & -0.40 & -0.01 & 0.04 & 0.02 & 0.82 & -0.38 & -0.15 & 0.10 & -0.01 \\
\hline Spikelet per spike & 0.45 & -0.19 & -0.05 & -0.07 & 0.35 & 0.36 & -0.15 & 0.09 & -0.69 \\
\hline Number of kernels per spike & 0.46 & -0.17 & -0.09 & -0.07 & 0.33 & 0.28 & -0.11 & 0.13 & 0.72 \\
\hline Thousand seed weight (gm) & -0.27 & 0.31 & 0.69 & 0.01 & 0.05 & 0.55 & -0.16 & 0.12 & 0.05 \\
\hline Grain yield (quintal ha ${ }^{-1}$ ) & 0.03 & -0.48 & 0.47 & -0.60 & 0.01 & -0.16 & 0.39 & -0.10 & 0.00 \\
\hline Eigenvalue & 3.87 & 2.22 & 0.75 & 0.71 & 0.49 & 0.42 & 0.37 & 0.17 & 0.01 \\
\hline Variability (\%) & 43.02 & 24.66 & 8.31 & 7.86 & 5.40 & 4.65 & 4.10 & 1.90 & 0.10 \\
\hline Cumulative $\%$ & 43.02 & 67.68 & 75.99 & 83.85 & 89.25 & 93.90 & 98.00 & 99.90 & 100.00 \\
\hline
\end{tabular}

Biplot was done based on the two PCs (Figure 1). The genotypes and the quantitative traits were shown on a biplot to visualize their associations. The first and the second PC biplot explained the $67.68 \%$ of total variability among the genotypes, displaying that number of kernel per spike, spikelet per spike and spike length days to $75 \%$ maturity and days to $50 \%$ flowering and grain yield number of kernel per spike, spikelet per spike and spike length ware considered as the most discriminating traits (Figure 1).

The genotypes positioned on the right top quadrant were closely associated and characterized by late flowering and maturity. The genotypes demarcated on the bottom right quadrant were associated highest grain yield, spikelet per spike and number of kernel per spike. The genotypes concentrated around the origin had similar genetic characteristics, while the genotypes that were found far from the origin are considered as unrelated genotypes (Figure 1). Therefore, the distribution of the genotype over the fourquadrant show that the presence of significant genetic diversity among the studied barley genotypes.

\subsection{Cluster Analysis}

The optimum number of clusters determined by the total within sum of square wss (Elbow Method) using R statistical software version 4.0.4. The distance matrix from nine quantitative traits was used to construct dendrograms based on the Unweighted Pair Group Method with Arithmetic Means (average).
The Euclidean distances of all possible pairs of 138 barley genotypes were estimated by Euclidean distance from nine quantitative traits and the results as Euclidean distance matrix. The Euclidean distances of the genotypes ranged from 0.61 to 8.06 with mean, standard deviation and coefficient of variation of $4.01,0.26$ and $6.4 \%$, respectively. The highest Euclidean distance was computed between accessions number 3689 and 3981 (8.06) accessions. Whereas, the lowest genetic distance was estimated between accession number 3767 and 3769 (0.61) (Figure 2).

Based on PC 1 and 2 scatter plot was constructed for four clusters (Figure 2). The scatter plot showed that the genotypes which have similar genetic makeup grouped in a cluster (near to overlap) and those genotypes have different genetics positioned in opposite corner of the scatter plot. However, the scatter plot shows only based on the variability of PC 1 and PC 2 means accommodate $67.7 \%$ of the variability.

The number of genotypes belonging to distinct clusters varied from one in fourth cluster IV to 65 in Cluster I. Cluster I was the largest cluster comprising of 65 genotypes (47.1\%) followed by cluster II with 48 genotypes (34.78\%) and cluster III with 24 genotypes (17.39\%) (Figure 3 and Table 2). Accession number 3689 was grouped in the fourth cluster alone. Clustering of the genotypes revealed ample variation for the agro-morphlogical traits and facilitated the selection of preferable landraces for pre-breeding and further evaluation. Several works have deployed cluster analysis to depict the heterogeneity and similarity among the accessions and interrelationship among characters. 


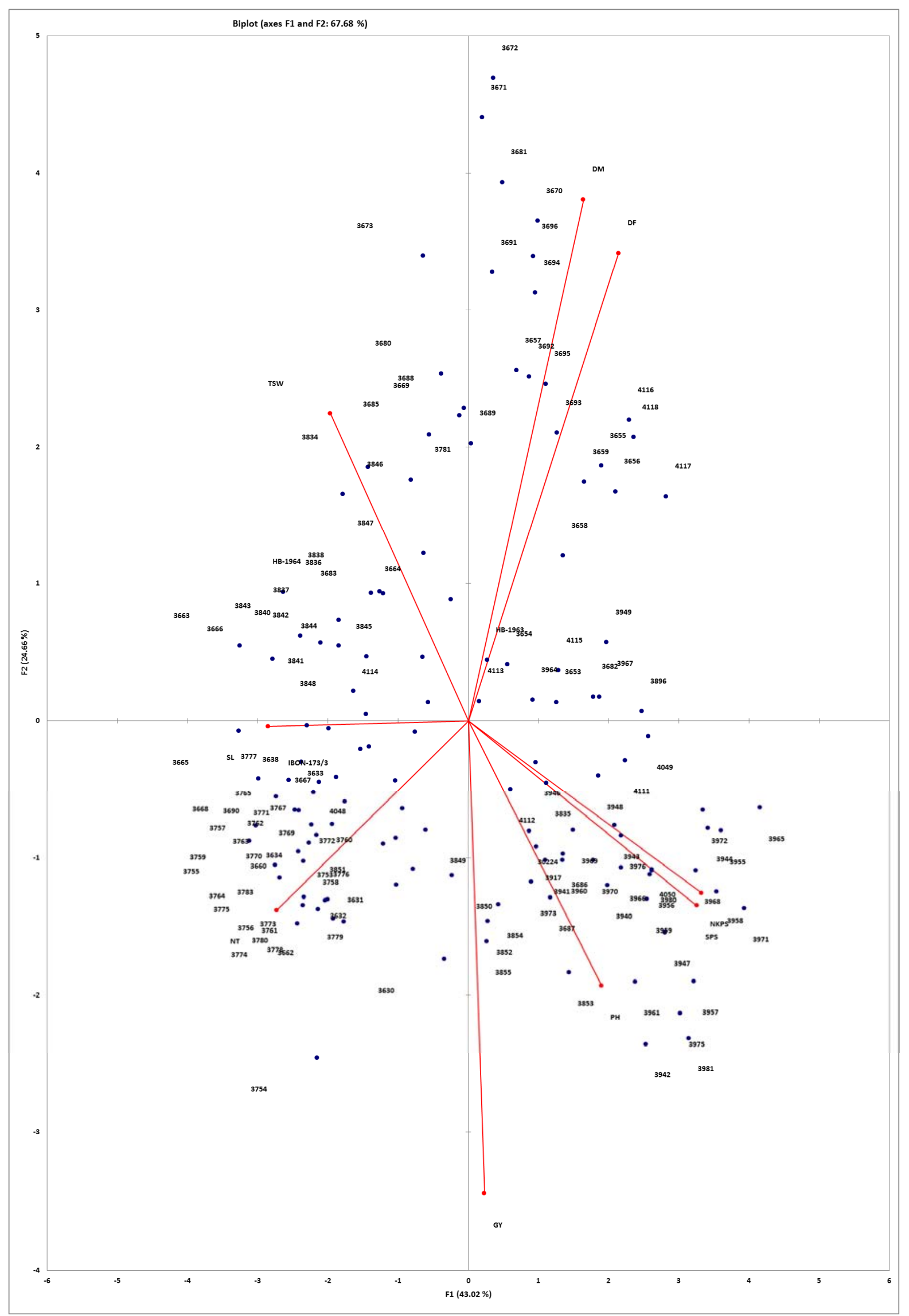

Figure 1. Biplot (axes PC1 and PC 2) of nine quantitative traits of 138 barley genotypes. 


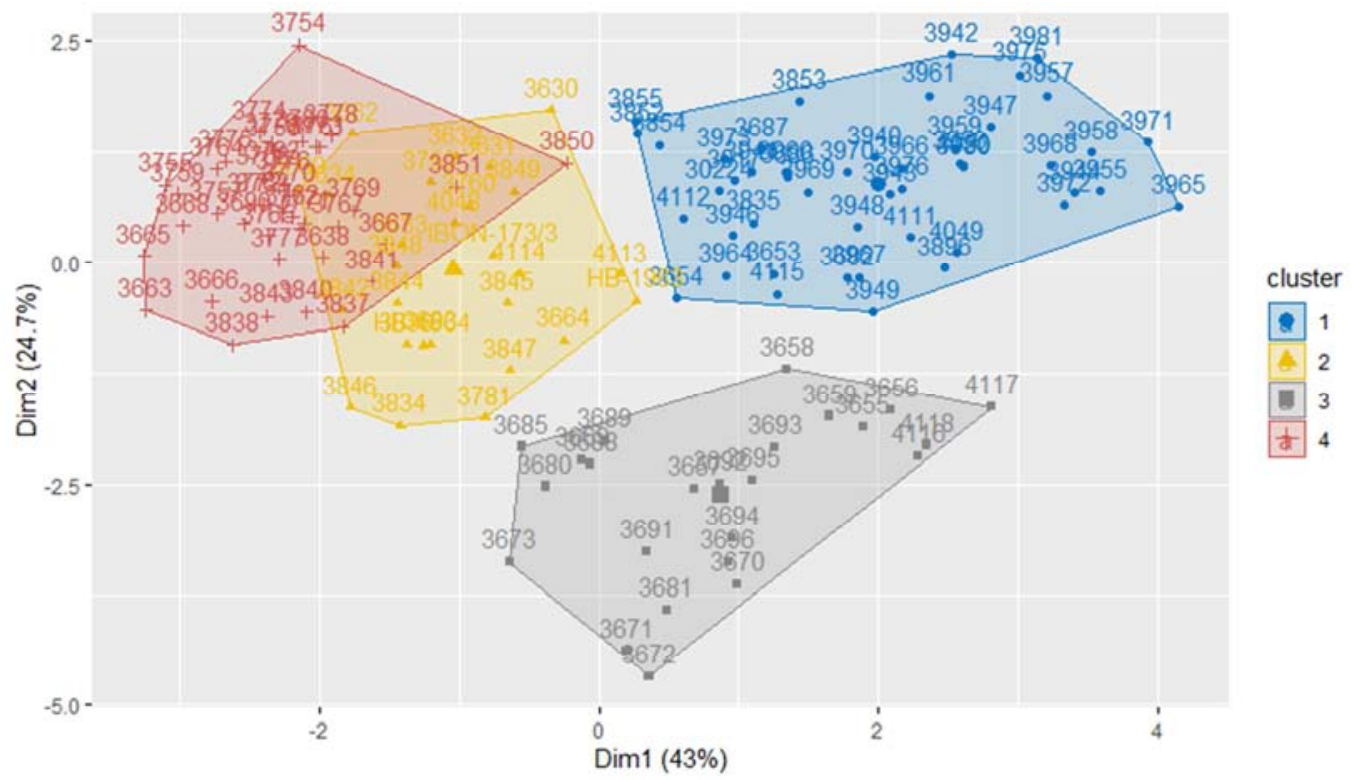

Figure 2. Scatter plot constructed based on PC1 and PC2 of nine quantitative traits for 138 barley genotypes.

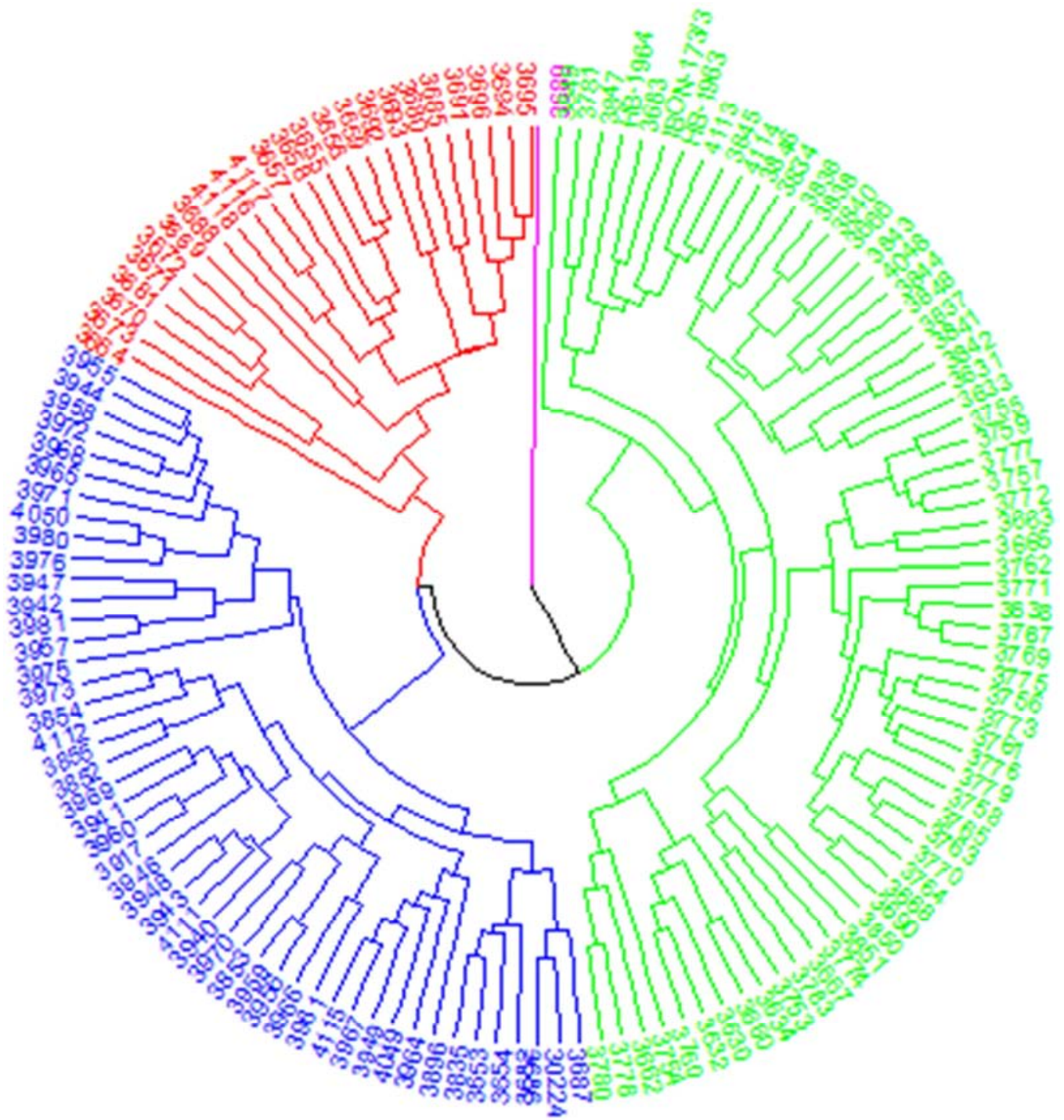

Figure 3. Dendrogram of barley genotypes based on nine quantitative traits, depicting genotypic relationships among accessions (average clustering methods). 
Table 2. Clusters, number of genotypes, list of genotypes in each cluster of 138 Barley genotypes.

\begin{tabular}{|c|c|c|}
\hline Clusters & Number of Genotypes & Lists of genotypes \\
\hline I & 65 & 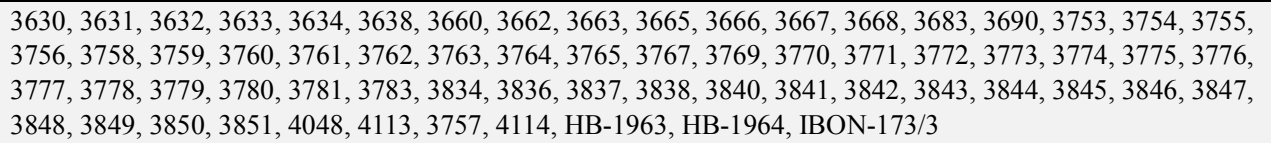 \\
\hline II & 48 & $\begin{array}{l}\text { 3653, 3654, 3682, 30224, 3686, 3687, 3835, 3852, 3853, 3854, 3855, 3896, 3917, 3940, 3941, 3942, 3943, 3944, } \\
\text { 3946, 3947, 3948, 3949, 3955, 3956, 3957, 3958, 3959, 3960, 3961, 3964, 3965, 3966, 3967, 3968, 3969, 3970, } \\
\text { 3971, 3972, 3973, 3975, 3976, 3980, 3981, 4049, 4050, 4111, 4112, 4115 }\end{array}$ \\
\hline III & 24 & $\begin{array}{l}3655,3656,3657,3658,3659,3664,36669,3670,3685,3671,3672,3673,3680,3681,3691,3692,3688,3672 \text {, } \\
3693,3694,3696,4116,4117,4118\end{array}$ \\
\hline IV & 1 & 3689 \\
\hline
\end{tabular}

In agreement with the current result, Mekonnon et al. [13], studied 102 Ethiopian barley farmer's varieties accessions and five standard checks and grouped these genotypes into five clusters. They found maximum genotype (44 genotypes) in cluster I and minimum genotypes (two genotypes) in cluster V. The rest of the genotypes were distributed in the remaining clusters ensuring the availability of genotypic variability. Addisu and Shumet [14] also studied 36 barley farmer's varieties accessions in southern Ethiopia and categorized them into four clusters. According to their study the maximum numbers of genotypes (11) were grouped in cluster II and IV and the minimum number were four in cluster II. These studies confirm that Ethiopian barley farmer's varieties have great genetic variability.
Genotypes clustered in the first cluster have the short days to flowering (52.78) days while genotypes grouped in cluster III have the longest days to flower which is 71.83 days. In addition, the highest mean of plant height of genotypes was recorded from cluster III. The result suggested that genotypes found in cluster I could be used for areas that have short period of rainfall. In contrast, genotypes clustered in cluster III suggested growing to areas of the country which have prolonged rainfall period. Genotypes grouped in cluster III are characterized by having the highest number of kernels per spike, and grain yield. The single genotypes grouped alone has the character of smallest grain yield and highest plant height. Here the reason for the small grain yield may be associated with lodging due to high plant height (Table 3).

Table 3. Cluster mean value for nine quantitative traits of 138 barley genotypes.

\begin{tabular}{llll}
\hline Traits & Cluster I & Cluster II & Cluster III \\
\hline Days to 50\% flowering & 52.78 & 60.42 & 71.83 \\
Days to 75\% maturity & 85.54 & 92.17 & 80.96 \\
Plant height (cm) & 83.05 & 92.83 & 120.33 \\
Number of tillers per plant & 5.57 & 4.44 & 4.38 \\
Spike length (cm) & 8.68 & 6.96 & 7.33 \\
Spikelet per spike & 23.49 & 39.71 & 29.07 \\
Number of kernels per spike & 21.35 & 38.82 & 28.45 \\
Thousand seed weight (gm) & 44.91 & 37.46 & 45.08 \\
Grain yield (quintal ha ${ }^{-1}$ ) & 32.22 & 35.69 & 124.00 \\
\hline
\end{tabular}

The clustering pattern indicated in the study revealed the existence of variability among the barley genotypes. Characterization of the genotypes and clustering them based on their morphological traits and genetic similarity will help in identification and selection of the best parents for hybridization. Therefore, grouping of genotypes by using multivariate methods of analysis based on their similarity in the present study would be valuable for barley breeders in that the most important accessions in the population may be selected from different clusters for barley improvement programs.

\section{Conclusion}

A total of one hundred thirty-eight accessions including three standard checks were evaluated for nine quantitative characters. The principal component analysis showed the variability of the accessions that the first principal component accounts for $43.02 \%$ and the second $24.66 \%$ of $67.68 \%$ of the total variability among the genotypes for traits considered for the study. Thus, the major quantitative traits contributing to variation among the studied genotypes include spike length, number of tillers per plant, days to $75 \%$ maturity and days to $50 \%$ flowering. Clustering patterns of the genotypes grouped them into 4 distinct clusters of different sizes ranging from 65 genotypes $(47.1 \%)$ in cluster $I$ to one genotype $(0.72 \%)$ in IV indicated that the existence of variability between barley genotypes that will help in identification and selection of the best parents. Generally, the accessions used in this study are diversified and offer an opportunity for plant breeders to conduct further breeding activities through selecting the most promising base populations of the crop accession or by crossing these divergent genotypes with each other focusing on major agronomic traits. 


\section{References}

[1] Harlan, J. R., 1969. Ethiopia: a center of diversity. Economic botany, 23 (4), pp. 309-314.

[2] Lakew, B., Semeane, Y., Alemayehu, F., Gebre, H., Grando, S., van Leur, J. A. and Ceccarelli, S., 1997. Exploiting the diversity of barley landraces in Ethiopia. Genetic Resources and Crop Evolution, 44 (2), pp. 109-116.

[3] (CSA, 2020) Central Statistical Agency (CSA), Agricultural Sample Survey, Report on Area and Production of Major Crops, CSA, Addis Ababa, Ethiopia, 2019.

[4] Shewayrga, H. and Sopade, P. A., 2011. Ethnobotany, diverse food uses, claimed health benefits and implications on conservation of barley landraces in North Eastern Ethiopia highlands. Journal of ethnobiology and ethnomedicine, 7 (1), pp. 1-15.

[5] Molla, M. B., 2015. Land use/land cover dynamics in the central rift valley region of Ethiopia: Case of Arsi Negele District. African Journal of Agricultural Research, 10 (5), pp. 434-449.

[6] IPGRI. 1994. Descriptors for barley (Hordium vulgare L.). International Plant Genetic Resources Institute, Rome, Italy.

[7] Aravind, J., Mukesh Sankar, S., Wankhede, D. P., and Kaur, V. (2021). Augmented RCBD: Analysis of Augmented Randomised Complete Block Designs. $\mathrm{R}$ package version 0.1.4, https://aravind-j.github.io/augmentedRCBD/https://cran.rproject.org/package=augmentedRCBD.

[8] Sebastien Le, Julie Josse, Francois Husson (2008). Facto Mine R: An R Package for Multivariate Analysis. Journal of Statistical Software, 25 (1), 1-18. 10.18637/jss.v025.i01

[9] Sneath, P. H. and Sokal, R. R. 1973. Numerical taxonomy. The principles and practice of numerical classification.

[10] Alboukadel Kassambara and Fabian Mundt (2020). factoextra: Extract and Visualize the Results of Multivariate Data Analyses. $\mathrm{R}$ package version 1.0.7. https://CRAN.Rproject.org/package $=$ factoextra

[11] Ebrahim, S., Shiferaw, E. and Hailu, F., 2015. Evaluation of genetic diversity in barley (Hordeum vulgare L.) from Wollo high land areas using agromorphological traits and hordein. African Journal of Biotechnology, 14 (22), pp. 1886-1896.

[12] Abera, K. T., 2009. Agronomic evaluation of Ethiopian barley (Hordeum vulgare L.) landrace populations under drought stress conditions in low-rainfall areas of Ethiopia.

[13] Mekonnon, B., Lakew, B. and Dessalegn, T., 2015. Morphological diversity and association of traits in Ethiopian food barley (Hordeum vulgare L.) landraces in relation to regions of origin and altitudes. Journal of Plant Breeding and Crop Science, 7 (2), pp. 44-54.

[14] Addisu, F. and Shumet, T., 2015. Variability, heritability and genetic advance for some yield and yield related traits in barley (Hordeum vulgare L.) landraces in Ethiopia. International Journal of Plant Breeding and Genetics, 9 (2), pp. 68-76. 